OPEN ACCESS

Edited by:

Eddie A. James,

Benaroya Research Institute,

United States

Reviewed by:

Vincent C. Lombardi,

University of Nevada, United States

Jonas J. W. Kuiper,

University Medical Center Utrecht,

Netherlands

*Correspondence:

Seung-Jung Kee

sjkee@jnu.ac.kr

Yong-Wook Park

parkyw@jnu.ac.kr

${ }^{+}$These authors have contributed equally to this work and

share first authorship

Specialty section:

This article was submitted to

Antigen Presenting Cell Biology,

a section of the journal

Frontiers in Immunology

Received: 26 April 2021 Accepted: 21 June 2021

Published: 01 July 2021

Citation:

Kang S-J, Park K-J, Jin H-M, Cho Y-N, Oh TH, Kim SE, Kim UJ, Park K-H, Jung S-I, Kim T-O, Kim HS, Jo Y-G, Ju JK, Kee S-J and Park Y-W (2021) Circulating Plasmacytoid and

Conventional Dendritic Cells Are Numerically and Functionally Deficient in Patients With Scrub Typhus.

Front. Immunol. 12:700755. doi: 10.3389/fimmu.2021.700755

\section{Circulating Plasmacytoid and Conventional Dendritic Cells Are Numerically and Functionally Deficient in Patients With Scrub Typhus}

\author{
Seung-Ji Kang ${ }^{1+}$, Ki-Jeong Park ${ }^{2+}$, Hye-Mi Jin ${ }^{2 \dagger}$, Young-Nan Cho ${ }^{2}$, Tae Hoon Oh ${ }^{1}$, \\ Seong Eun Kim ${ }^{1}$, Uh Jin Kim ${ }^{1}$, Kyung-Hwa Park ${ }^{1}$, Sook-In Jung ${ }^{1}$, Tae-Ok Kim ${ }^{3}$, \\ Hyo Shin Kim ${ }^{4}$, Young-Goun Jo ${ }^{4}$, Jae Kyun Ju ${ }^{4}$, Seung-Jung Kee ${ }^{5 *}$ \\ and Yong-Wook Park ${ }^{2 *}$ \\ 1 Department of Infectious Diseases, Chonnam National University Medical School and Hospital, Gwangju, South Korea,
2 Department of Rheumatology, Chonnam National University Medical School and Hospital, Gwangju, South Korea,
${ }^{3}$ Department of Pulmonology, Chonnam National University Medical School and Hospital, Gwangju, South Korea,
${ }^{4}$ Department of Surgery, Chonnam National University Medical School and Hospital, Gwangju, South Korea,
${ }^{5}$ Department of Laboratory Medicine, Chonnam National University Medical School and Hospital, Gwangju, South Korea
}

Background: Dendritic cells (DCs) are specialized antigen-presenting cells known to bridge innate and adaptive immune reactions. However, the relationship between circulating DCs and Orientia tsutsugamushi infection is unclear. Therefore, this study aimed to examine the level and function of plasmacytoid DCs (pDCs) and conventional DCs (cDCs), two subsets of circulating DCs, in scrub typhus patients.

Methods: The study included 35 scrub typhus patients and 35 healthy controls (HCs). pDC and cDC levels, CD86 and CD274 expression, and cytokine levels were measured using flow cytometry.

Results: Circulating pDC and cDC levels were found to be significantly reduced in scrub typhus patients, which were correlated with disease severity. The patients displayed increased percentages of $\mathrm{CD}^{+} 6^{+} \mathrm{pDCs}, \mathrm{CD} 274^{+} \mathrm{pDCs}$, and $\mathrm{CD} 274^{+} \mathrm{cDCs}$ in the peripheral blood. The alterations in the levels and surface phenotypes of pDCs and cDCs were recovered in the remission state. In addition, the production of interferon (IFN)- $\alpha$ and tumor necrosis factor (TNF)- $\alpha$ by circulating pDCs, and interleukin (IL)-12 and TNF- $\alpha$ by circulating cDCs was reduced in scrub typhus patients. Interestingly, our in vitro experiments showed that the percentages of $\mathrm{CD}^{+} 6^{+} \mathrm{pDCs}, \mathrm{CD} 274^{+} \mathrm{pDCs}$, and CD274 CDCs were increased in cultures treated with cytokines including IFN- $\gamma, \mathrm{IL}-12$, and TNF- $\alpha$.

Conclusions: This study demonstrates that circulating pDCs and cDCs are numerically deficient and functionally impaired in scrub typhus patients. In addition, alterations in the 
expression levels of surface phenotypes of pDCs and cDCs could be affected by proinflammatory cytokines.

Keywords: cytokine, Orientia tsutsugamushi, plasmacytoid dendritic cells, scrub typhus, conventional dendritic cells.

\section{INTRODUCTION}

Orientia tsutsugamushi is an obligate intracellular bacterium that causes scrub typhus, a febrile illness widespread across the world (1). The disease initially exhibits typical eschar, rash, and if not managed sufficiently, fatal conditions including acute kidney injury, liver failure, meningoencephalitis, and multiple organ failure can develop $(2,3)$. Approximately a million patients are diagnosed each year in a broad area from the Asian-Pacific region, the so-called "Tsutsugamushi Triangle", to Africa, Europe, and South America (1). Furthermore, it is spreading from rural to urban areas, which raises considerable concern in endemic countries (4).

Though the exact pathophysiology remains unclear, $O$. tsutsugamushi induces a range of dysregulated immune responses (5). The pathogen invades endothelial cells (ECs), monocytes, and dendritic cells (DCs), which are activated to secrete various cytokines and chemokines, provoking Th1 and Th2 dysregulation and the functional impairment of $\mathrm{T}$ lymphocytes (6-9). Recent investigations on unconventional immune cells such as mucosal-associated invariant T (MAIT) cells, natural killer (NK) cells, and natural killer T (NKT) cells suggest variations in frequency and function along with clinical relevance to the disease (10-12). Among these antigen-presenting cells (APCs), DCs are the most potent, central, and professional component that initiates and orchestrates immune reactions at the interface between innate and adaptive immunity (13). Currently, DCs can be classified into different subsets of conventional DCs (cDCs, formerly myeloid DC), plasmacytoid DCs (pDCs), monocyte-derived DCs (moDCs), and Langerhans cells based on their surface phenotype and functions (13-15). Of these different DCs, cDCs and pDCs are two main subsets of naturally occurring DCs that circulate in the peripheral blood. pDCs release type I interferon (IFN) against viruses, produce proinflammatory cytokines, and express major histocompatibility class (MHC) class II antigens and co-stimulatory molecules that activate numerous immune cells, including NK cells and NKT cells (16). cDCs are potent and specialized activators of $\mathrm{T}$ cells (13).

Several studies have described the relevance of DCs to scrub typhus. In one murine model, O. tsutsugamushi evaded autophagy and effectively invaded bone marrow-derived DCs, which showed impaired maturation and migration into lymphatic tissues (17). Another research study using human moDCs, a distinct DC population that matures during inflammation, reported that the pathogen replicated in moDCs, provoked the maturation of the cells, and triggered the secretion of cytokines, consequently stimulating $\mathrm{CD} 4{ }^{+} \mathrm{T}$ cells (18). However, a study on the levels and functions of pDCs and cDCs in scrub typhus has yet to be conducted. Therefore, this study aimed to examine the levels and functions of pDCs and cDCs in scrub typhus, evaluate their clinical relevance, and investigate their roles under inflammatory conditions.

\section{MATERIALS AND METHODS}

\section{Study Subjects}

The study cohort was comprised of 35 patients with scrub typhus (20 women and 15 men; mean age \pm SD, $65.6 \pm 15.4$ years) and 35 healthy controls (HCs; 25 women and 10 men; mean age \pm $\mathrm{SD}, 37.1 \pm 7.5$ years). The diagnosis of scrub typhus was performed by detecting O. tsutsugamushi antibodies in the patient's serum using a passive hemagglutination assay kit (Genedia Tsutsu PHA II Test Kit; GreenCross SangA, Yongin, Korea). A positive result was defined as a titer of $\geq 1: 80$ in a single serum sample or at least a 4-fold rise in antibody titer at a followup examination, as described previously $(10-12,19)$. According to the number of dysfunctional organs, scrub typhus was graded into severe ( $\geq 2$ organ dysfunctions), moderate (one organ dysfunction), and mild disease (no organ dysfunction) as previously described (20). The definition of organ dysfunction was: (1) renal dysfunction, creatinine $\geq 2.5 \mathrm{mg} / \mathrm{dL}$; (2) hepatic dysfunction, total bilirubin $\geq 2.5 \mathrm{mg} / \mathrm{dL}$; (3) pulmonary dysfunction, bilateral pulmonary infiltration on chest X-rays with moderate to severe hypoxia $\left(\mathrm{PaO}_{2} / \mathrm{FiO}_{2}<300 \mathrm{mmHg}\right.$ or $\mathrm{PaO}_{2}<60 \mathrm{mmHg}$ or $\mathrm{SpO}_{2}<90 \%$ ); (4) cardiovascular dysfunction, systolic blood pressure $<80 \mathrm{mmHg}$ despite fluid resuscitation; and (5) central nervous system dysfunction, significantly altered sensorium with a Glasgow Coma Scale (GCS) score of eight out of 15. All HCs were recruited in the same area (Jeollanam-do, South Korea) as the patients resided. HCs had no severe comorbidity such as malignancy, chronic liver, pulmonary, renal diseases, autoimmune disease, or fever within 72 hours prior to enrollment.

\section{Monoclonal Antibodies and Flow Cytometry}

The following monoclonal antibodies (mAbs) and reagents were used in this study: fluorescein isothiocyanate (FITC)-conjugated Lineage Cocktail 1(CD3, CD14, CD16, CD19, CD20, CD56), phycoerythrin (PE)-conjugated anti-CD123, anti-CD86, and anti-CD274; allophycocyanin (APC)-conjugated anti-CD11c, anti-CD86, and anti-CD274; BV421-conjugated anti-HLA-DR; Alexa Fluor 647-conjugated anti-IFN- $\alpha$; PE-conjugated anti- 
interleukin-12 (anti-IL-12), PE-Cy7-conjugated anti-tumor necrosis factor- $\alpha$ (anti-TNF- $\alpha$ ) mAb, and PE-conjugated mouse IgG isotype control (all from Becton Dickinson, San Diego, CA, USA). The cells were stained with combinations of the appropriate $\mathrm{mAbs}$ for 20 minutes at $4^{\circ} \mathrm{C}$. The stained cells were analyzed on a Navios flow cytometer using Kaluza software (version 1.5a; Beckman Coulter, Brea, CA, USA).

\section{Isolation of Peripheral Blood Mononuclear Cells (PBMCs) and Identification of pDCs and cDCs}

Peripheral venous blood samples were collected in heparincontaining tubes, and PBMCs were isolated by density-gradient centrifugation using Ficoll-Paque Plus solution (Amersham Biosciences, Uppsala, Sweden). pDCs and cDCs were identified phenotypically as Lin1 ${ }^{-} \mathrm{HLA}-\mathrm{DR}^{+} \mathrm{CD} 123^{+}$cells and Lin1 ${ }^{-} \mathrm{HLA}-$ $\mathrm{DR}^{++} \mathrm{CD} 11 \mathrm{c}^{+}$cells by flow cytometry, as previously described (21).

\section{Intracellular Cytokine Staining}

Freshly isolated PBMCs $\left(1 \times 10^{6} /\right.$ well $)$ were incubated in $1 \mathrm{~mL}$ of complete media, consisting of RPMI 1640, $2 \mathrm{mM}_{\mathrm{L}}$-glutamine, $100 \mathrm{units} / \mathrm{mL}$ of penicillin, and $100 \mu \mathrm{g} / \mathrm{mL}$ of streptomycin, and supplemented with $10 \%$ fetal bovine serum (FBS) for 2 hours in the presence of $10 \mu \mathrm{g} / \mathrm{mL}$ CpG (ODN2336; InvivoGen, San Diego, CA, USA) or $10 \mu \mathrm{g} / \mathrm{mL}$ non-CpG ODN control (InvivoGen) to stimulate pDCs and in the presence of $10 \mathrm{ng} /$ $\mathrm{mL}$ IFN- $\gamma$ (PeproTech, London, UK) and $2 \mu \mathrm{g} / \mathrm{mL}$ lipopolysaccharide (LPS; Sigma-Aldrich, St. Louis, MO, USA) to stimulate cDCs. For intracellular cytokine staining, $1 \mu \mathrm{L}$ of brefeldin A (GolgiPlug; BD Biosciences, San Diego, CA, USA) was added for each $1 \mathrm{~mL}$ of cell culture. After incubation for an additional 4 hours, the cells were stained with FITC-conjugated Lineage Cocktail 1, PE-conjugated anti-CD123, APC-conjugated anti-CD11c, and BV421-conjugated anti-HLA-DR mAbs for 20 minutes at $4^{\circ} \mathrm{C}$, fixed in $4 \%$ paraformaldehyde for 15 minutes at room temperature, and permeabilized with Perm/Wash solution (BD Biosciences) for 10 minutes. The cells were then stained with Alexa Fluor 647-conjugated anti-IFN- $\alpha$, PE-conjugated anti-IL12 , and PE-Cy7-conjugated anti-TNF- $\alpha$ mAbs for 30 minutes at $4^{\circ} \mathrm{C}$ and analyzed by flow cytometry.

\section{Statistical Analysis}

All comparisons of percentages, absolute numbers, cytokine levels, and expression levels of CD86 and CD274 in pDCs and cDCs were performed by analysis of covariance after adjusting for age and sex using the Bonferroni correction for multiple comparisons (ANCOVA). The Wilcoxon matched-pairs signed-rank test was used to compare changes in the cell numbers and expression of $\mathrm{pDC}$ and $\mathrm{CDC}$ phenotypes according to disease activity. To compare changes in surface phenotypes of pDCs and cDCs treated by pro-inflammatory cytokines, Kruskal-Wallis analysis with Dunn's post hoc test was used for multiple comparisons. $P$-values of less than 0.05 were considered statistically significant. Statistical analysis was performed and graphs were generated using SPSS version 26.0 software (SPSS, Chicago, IL, USA) and GraphPad Prism

version 5.03 software (GraphPad Software, San Diego, CA, USA), respectively.

\section{RESULTS}

\section{Subject Characteristics}

The clinical and laboratory characteristics of 35 patients with scrub typhus are summarized in Table 1. According to disease severity based on the number of organs with dysfunction in these patients, $16(45.7 \%), 12(34.3 \%)$, and $7(20.0 \%)$ had mild, moderate, and severe disease, respectively. Longitudinal monitoring was performed for 20 patients from the active state (before antibiotic therapy) to the remitted state (defined as resolution of all presenting symptoms after antibiotic therapy). Nine of those patients were available for measuring cell levels and surface phenotypes of circulating pDCs and cDCs.

TABLE 1 | Clinical and laboratory characteristics of 35 patients with scrub typhus.

Variables

Age, years, mean $\pm S D$

Male/female, $\mathrm{n}$

Clinical variables, $\mathrm{n}(\%)$

Fever

Rash

Eschar

Confusion

Severity of disease, $n$ (\%)

Mild disease

Moderate disease

Severe disease

Organ dysfunction, n (\%)

Renal dysfunction

Hepatic dysfunction

CNS dysfunction

Respiratory dysfunction

Circulatory dysfunction

Comorbid conditions, n (\%)

Diabetes mellitus

Cardiovascular disease

Chronic kidney disease

Chronic hepatic disease

Chronic lung disease

Malignancy

Laboratory variables, mean $\pm \mathrm{SD}$

Leukocyte count, cells $/ \mu \mathrm{L}$

Lymphocyte count, cells/ $\mu \mathrm{L}$

Hemoglobin level, g/dL

Neutrophil count, cells $/ \mu \mathrm{L}$

Platelet count, $\times 10^{3}$ cells $/ \mu \mathrm{L}$

Total bilirubin level, mg/dL

Total protein level, $\mathrm{g} / \mathrm{dL}$

Albumin level, g/dL

AST level, U/L

ALT level, U/L

Alkaline phosphatase level, U/L

LDH level, U/L

CRP level, mg/dL

Time at hospital visit ${ }^{a}$, days, mean \pm SD

ALT, alanine aminotransferase: AST, $C R P, C$-reactive protein; $L D H$, lactate dehydrogenase; $n$, number; $S D$, standard deviation.

${ }^{a}$ Time from symptom onset to antibiotic therapy.

Scrub typhus

$65.6 \pm 15.4$

$15 / 20$

31 (88.6)

$24(68.6)$

$28(80.0)$

$5(14.3)$

$16(45.7)$

$12(34.3)$

7 (20.0)

$2(5.71)$

4 (11.4)

$4(11.4)$

$14(40.0)$

7 (20.0)

$8(22.9)$

$3(8.57)$

$1(2.86)$

$1(2.86)$

$0(0.00)$

$2(5.71)$

$7760 \pm 3416$

$2109 \pm 1169$

$11.7 \pm 1.7$

$5008 \pm 3198$

$173 \pm 99$

$0.9 \pm 0.6$

$6.0 \pm 0.7$

$2.9 \pm 0.6$

$159 \pm 178$

$122 \pm 200$

$192 \pm 137$

$908 \pm 276$

$10.4 \pm 8.4$

$6.1 \pm 3.3$ 


\section{Reduced Numbers of pDCs and cDCs in Scrub Typhus Patients}

The percentage and absolute numbers of pDCs and cDCs in the peripheral blood samples of 35 scrub typhus patients and $35 \mathrm{HCs}$ were determined by flow cytometry. pDCs and cDCs were defined as Lin $1^{-} \mathrm{HLA}-\mathrm{DR}^{+} \mathrm{CD} 123^{+}$cells and Lin $1^{-} \mathrm{HLA}-$ $\mathrm{DR}^{++} \mathrm{CD} 11 \mathrm{c}^{+}$cells, respectively (Figures $\mathbf{1 A}, \mathbf{D}$ ). The percentage of circulating $\mathrm{pDCs}$ and $\mathrm{cDCs}$ were significantly lower in the patients than in the HCs (for pDCs, median $0.01 \%$ versus $0.17 \%, P<0.0001$; for cDCs, median $0.01 \%$ versus $0.12 \%, P=0.001$; Figures 1 B, E). The absolute numbers of pDCs and $\mathrm{cDCs}$ were calculated by multiplying the $\mathrm{pDC}$ and $\mathrm{cDC}$ fractions in the mononuclear cells gate (using a flow cytometer) by absolute PBMC count (per milliliter of peripheral blood) determined using a standard hemocytometer. Scrub typhus patients had significantly lower absolute numbers of pDCs and cDCs than the HCs (for pDCs, median 267 cells $/ \mathrm{mL}$ versus 3744 cells $/ \mathrm{mL}, P=0.007$; for $\mathrm{cDCs}$, median 259 cells $/ \mathrm{mL}$ versus 3170 cells $/ \mathrm{mL}, P<0.0001$; Figures 1C, F).

\section{Relationship Between pDC and cDC Levels and Clinical Parameters in Scrub Typhus Patients}

To evaluate the clinical relevance of pDC and cDC levels in 35 patients with scrub typhus, we investigated the correlation between $\mathrm{pDC}$ and $\mathrm{cDC}$ percentages in the peripheral blood and clinical parameters by Spearman's rank correlation analysis. The correlation results revealed that both circulating $\mathrm{pDC}$ and $\mathrm{cDC}$ percentages were significantly correlated with albumin levels ( $P=0.003$ and $P=0.019$, respectively) and disease severity $(P=0.038$ and $P=0.034$, respectively). However, our experiments showed no significant correlation between circulating $\mathrm{pDC}$ and $\mathrm{cDC}$ percentages and leukocyte count, lymphocyte count, hemoglobin level, neutrophil count, platelet count, total bilirubin level, total protein level, aspartate aminotransferase level, alanine aminotransferase level, alkaline phosphatase level, lactate dehydrogenase level, or C-reactive protein level (Table 2).

\section{Activity of pDCs and cDCs in Scrub Typhus Patients}

DCs can either induce or regulate immune reactions using specific molecules (14). Among those molecules, CD86 is known as a co-stimulatory marker that activates $\mathrm{T}$ cells, whereas CD274 is a co-inhibitory marker that restricts $\mathrm{T}$ cell function $(14,22)$. To examine the activity of both pDCs and cDCs, the expression levels of CD86 and CD274 molecules by each DC subset were compared between 22 scrub typhus patients and $16 \mathrm{HCs}$ by flow cytometry. The percentages of both CD86 ${ }^{+}$ and $\mathrm{CD} 274^{+}$pDCs were significantly higher in scrub typhus patients compared to the HCs (for CD86 ${ }^{+}$pDCs, median $39.9 \%$ versus $11.3 \%, P=0.031$; and for $\mathrm{CD}_{2} 74^{+} \mathrm{pDCs}, 13.6 \%$ versus $0.2 \%, P=0.001$; Figures $2 \mathrm{~A}, \mathbf{B}$ ). The percentages of CD $274^{+}$ cDCs were significantly higher in scrub typhus patients than in the HCs (median $17.0 \%$ versus $2.9 \%, P=0.006$; Figure 2D).
A

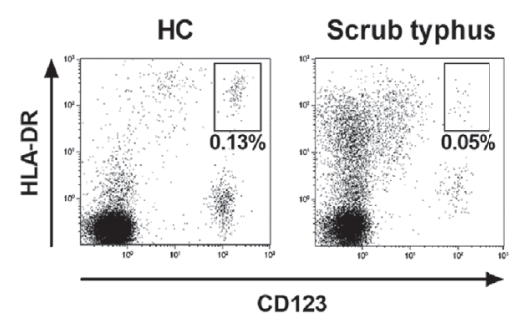

D

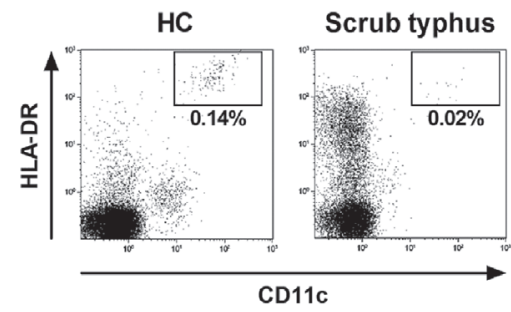

B

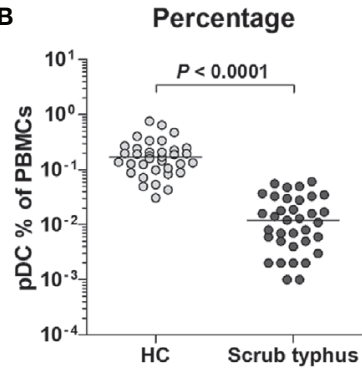

E

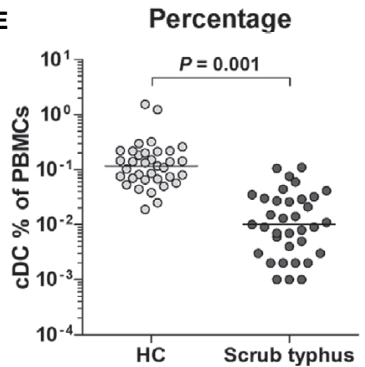

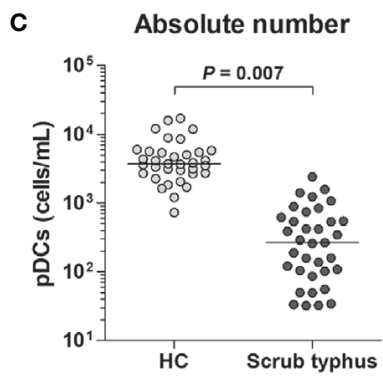

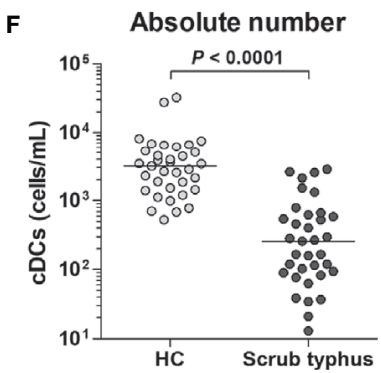

FIGURE 1 | Decreased numbers of circulating pDCs and cDCs in peripheral blood samples of scrub typhus patients. Freshly isolated PBMCs from 35 HCs and 35 scrub typhus patients were stained with APC-conjugated anti-CD11c, BV421-conjugated anti-HLA-DR, FITC-conjugated Lineage Cocktail 1, and PE-conjugated antiCD123 mAbs and then analyzed by flow cytometry. The percentages of pDCs and CDCs were calculated using a large gate including lymphocytes and monocytes. (A, D) Representative percentages of pDCs and cDCs determined by flow cytometry. (B, E) Percentages of pDCs and cDCs in PBMCs. (C, F) Absolute numbers of pDCs and cDCs (per milliliter of blood). The symbols represent individual subjects and the horizontal lines are median values. $P$ values were calculated by ANCOVA test. 
TABLE 2 | Spearman's correlation coefficients for the percentages of pDCs and cDCs with respect to clinical and laboratory parameters in 35 patients with scrub typhus.

\begin{tabular}{|c|c|c|c|c|}
\hline \multirow[t]{2}{*}{ Variable } & \multicolumn{2}{|c|}{ pDCs } & \multicolumn{2}{|c|}{ cDCs } \\
\hline & $\rho$ & $P$ value & $\rho$ & $P$ value \\
\hline Age (years) & -0.215 & 0.215 & -0.152 & 0.383 \\
\hline Leukocyte count (cells/ $\mu \mathrm{L})$ & 0.030 & 0.863 & -0.013 & 0.942 \\
\hline Lymphocyte count (cells/ $\mu \mathrm{L}$ ) & 0.087 & 0.621 & -0.045 & 0.796 \\
\hline Hemoglobin level (g/dL) & 0.255 & 0.140 & -0.038 & 0.827 \\
\hline Neutrophil count (cells/ $\mu \mathrm{L})$ & 0.028 & 0.871 & -0.044 & 0.802 \\
\hline Platelet count $\left(\times 10^{3}\right.$ cells $\left./ \mu \mathrm{L}\right)$ & -0.106 & 0.545 & -0.017 & 0.922 \\
\hline Total bilirubin level (mg/dL) & -0.136 & 0.459 & -0.085 & 0.643 \\
\hline Total protein level (g/dL) & 0.303 & 0.098 & 0.097 & 0.604 \\
\hline Albumin level (g/dL) & 0.509 & $0.003^{\star}$ & 0.411 & $0.019^{\star}$ \\
\hline AST level (U/L) & -0.258 & 0.147 & -0.087 & 0.632 \\
\hline ALT level (U/L) & 0.089 & 0.623 & 0.280 & 0.114 \\
\hline Alkaline phosphatase level (U/L) & -0.146 & 0.418 & -0.098 & 0.586 \\
\hline LDH level (U/L) & 0.026 & 0.900 & 0.028 & 0.895 \\
\hline CRP level (mg/dL) & 0.115 & 0.511 & -0.171 & 0.326 \\
\hline Severity & -0.358 & $0.038^{*}$ & -0.364 & $0.034^{*}$ \\
\hline
\end{tabular}

$A L T$, alanine aminotransferase; AST, aspartate aminotransferase; CRP, C-reactive protein; $L D H$, lactate dehydrogenase; $c D C s$, conventional dendritic cells; $p D C s$, plasmacytoid dendritic cells; $\rho$, correlation coefficient.

*Indicates statistical significance.

However, no significant difference was observed in $\mathrm{CD}^{+} 6^{+} \mathrm{cDC}$ percentages between the patients and HCs (Figure 2C).

\section{Impaired Cytokine Production in pDCs and cDCs From Scrub Typhus Patients}

We next measured the levels of representative cytokines secreted by $\mathrm{pDCs}$ and $\mathrm{cDCs}$ from scrub typhus patients. The PBMCs from 15 scrub typhus patients and 15 HCs were incubated for 2 hours in the presence of $\mathrm{CpG}$ (for pDCs stimulation) or IFN- $\gamma$ and LPS (for cDCs stimulation) and the expression levels of IFN$\alpha$, IL-12, and TNF- $\alpha$ in the $\mathrm{pDC}$ and $\mathrm{CDC}$ populations were examined at the single-cell level by intracellular flow cytometry (Figures 3A, C). The percentages of IFN- $\alpha^{+}$and TNF- $\alpha^{+}$pDCs were found to be significantly lower in scrub typhus patients than in $\mathrm{HCs}$ (for IFN- $\alpha^{+}$cells, median $1.0 \%$ versus $23.2 \%, P=0.001$; for TNF- $\alpha^{+}$cells, median $2.8 \%$ versus $22.0 \%, P=0.005$; Figure 3B). In addition, the percentages of IL- $12^{+}$and TNF- $\alpha^{+}$ cDCs were found to be significantly lower in scrub typhus patients compared to $\mathrm{HCs}$ (for IL- $12^{+}$cells, median $9.3 \%$ versus $28.8 \%, P=0.003$; for TNF- $\alpha^{+}$cells, median $24.7 \%$ versus $49.0 \%, P=0.019$; Figure 3D).

\section{Changes in Levels and Surface Phenotypes of Circulating pDCs and cDCs According to Disease Activity}

Based on our observation that the percentage of pDCs and cDCs was reduced during the active state of scrub typhus but the expression of CD86 and CD274 on pDCs and CD274 on cDCs was increased, we investigated whether changes in the proportion and surface phenotypes of pDCs and $\mathrm{cDCs}$ were related to disease activity. We found that the percentage of both pDCs and cDCs was greater when the disease was in remission than when it was active (for pDCs, median $0.13 \%$ versus $0.01 \%$, $P<0.0001$; for cDCs, median $0.12 \%$ versus $0.01 \%, P=0.0002$;
Figures $4 A, D)$. In the surface phenotypes, the percentages of CD86-expressing pDCs, and CD274-expressing pDCs and cDCs were lower in the remission state than in the active state (for $\mathrm{CD} 6^{+} \mathrm{pDCs}$, median $44.3 \%$ versus $50.0 \%, P=0.02$; for CD $274^{+}$ pDCs, median $8.4 \%$ versus $25.0 \%, P=0.0024$; for $\mathrm{CD} 274^{+} \mathrm{cDCs}$, median $6.1 \%$ versus 20.0\%, $P=0.004$; Figures 4B, C, F). Conversely, the percentage of CD86-expressing cDCs was higher in the remission state compared to the active state (median $98.5 \%$ versus $88.4 \%, P=0.014$; Figure $4 \mathrm{E}$ ).

\section{Effect of Stimulation With Pro- Inflammatory Cytokine Cocktail on the Activation of $\mathrm{pDCs}$ and $\mathrm{cDCs}$}

To determine whether pDCs and cDCs could be activated by pro-inflammatory cytokines, PBMCs from six HCs were incubated for 24 hours in the presence or absence of cytokine inhibitors (i.e., blocking antibodies against a cocktail of IFN- $\gamma$, IL-12, and TNF- $\alpha$ ) and then stimulated with a cytokine cocktail consisting of IFN- $\gamma$, IL-12, and TNF- $\alpha$ for 16 hours. KruskalWallis analysis showed signficant differences among baseline, cytokine-treated, and blocking antibody-treated groups for the expression of CD86 $(P=0.0135)$ or CD274 $(P=0.0023)$ in pDCs and CD274 $(P=0.0227)$ in $\mathrm{cDCs}$, except for the expression of CD86 $(P=0.1443)$ in cDCs. In addition, Dunn's post hoc test was used for correction for multiple comparisons to analyze the differences between baseline and cytokine-treated groups or between cytokine-treated and blocking antibody-treated groups. The percentages of $\mathrm{CD} 86^{+} \mathrm{pDCs}$ were found to be significantly higher in cytokine-treated cultures compared to cytokine-untreated cultures (median $10.4 \%$ versus $2.3 \%, P=$ 0.0067 ), and then normalized to the untreated levels after treatment with blocking antibodies (median 10.4\% versus 2.2, $P=0.0198$; Figure 5A). The percentages of $\mathrm{CD}_{274^{+}} \mathrm{pDCs}$ significantly increased in cytokine-treated cultures compared to cytokine-untreated cultures (median $11.8 \%$ versus $0.8 \%, P=$ 0.0045 ), and then normalized to the untreated levels after treatment with blocking antibodies (median $11.8 \%$ versus $0.01 \%, P=0.0015$; Figure 5B). The percentages of CD274 cDCs significantly increased in cytokine-treated cultures compared to cytokine-untreated cultures (median $32.8 \%$ versus $6.7 \%, P=0.0326$ ), and then normalized to the untreated levels after treatment with blocking antibodies (median $32.8 \%$ versus $3.2 \%, P=0.0102$; Figure 5D). However, the percentage of CD86 ${ }^{+}$ cDCs remained unchanged regardless of cytokine stimulation or cytokine blocking (Figure 5C).

\section{DISCUSSION}

To the best of our knowledge, this was the first study to examine the levels and functions of pDCs and cDCs and assess the clinical relevance in scrub typhus patients. The patients exhibited decreased percentages and absolute numbers of circulating pDCs and cDCs, which was correlated with disease severity, and they had increased percentages of $\mathrm{CD}^{+} 6^{+}$pDCs, CD $274^{+}$ pDCs, and $\mathrm{CD}_{2} 74^{+} \mathrm{cDCs}$ in the peripheral blood. These 
A

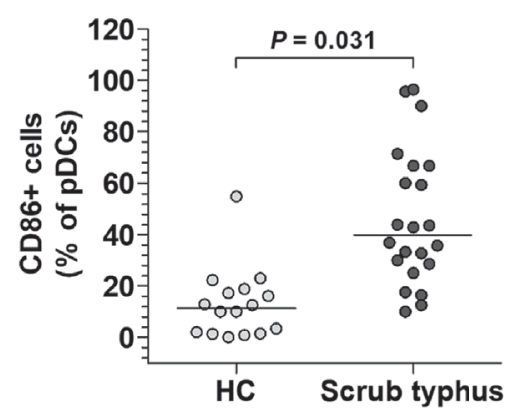

C

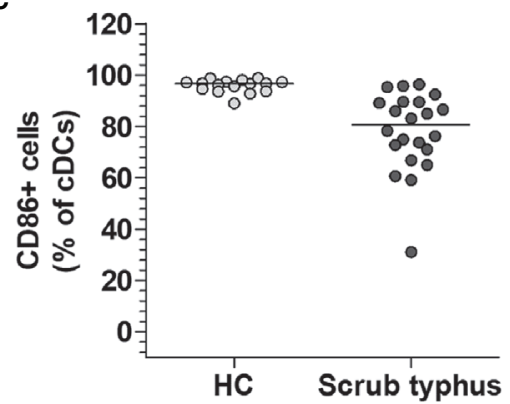

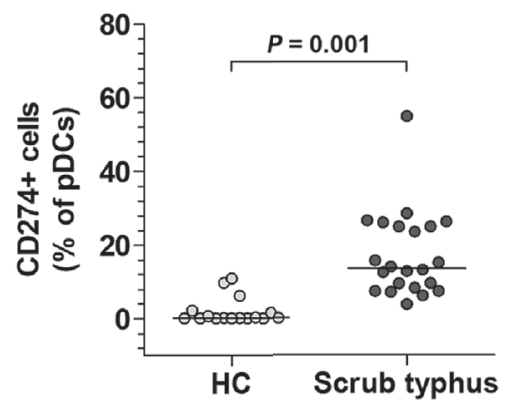

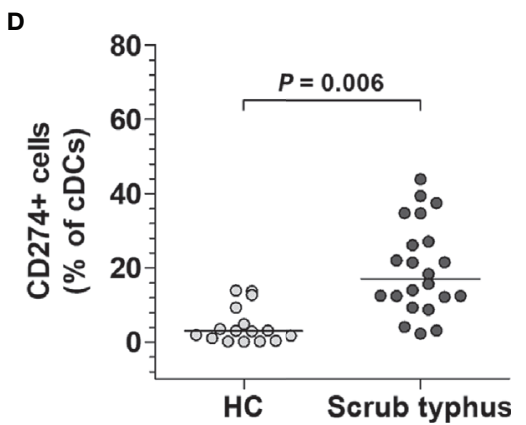

FIGURE 2 | Phenotypes of circulating pDCs and CDCs in scrub typhus patients. Freshly isolated PBMCs were stained with APC-conjugated anti-CD11c, anti-CD86, and anti-CD274; BV421-conjugated anti-HLA-DR; FITC-conjugated Lineage Cocktail 1; and PE-conjugated anti-CD86, anti-CD123, and anti-CD274 mAbs and then analyzed by flow cytometry. (A, C), Percentages of CD86-expressing pDCs and CDCs. (B, D), Percentages of CD274-expressing pDCs and cDCs. The data were obtained from $16 \mathrm{HCs}$ and 22 scrub typhus patients. The symbols represent individual subjects and the horizontal lines are median values. $P$ values were calculated by ANCOVA test.

alterations in the levels and surface phenotypes of pDCs and $\mathrm{cDCs}$ were recovered in the remission state. In addition, the production of IFN- $\alpha$ and TNF- $\alpha$ by circulating pDCs, and IL-12 and TNF- $\alpha$ by circulating cDCs was reduced in scrub typhus patients. Interestingly, our in vitro experiments showed that the activation of $\mathrm{pDCs}$ and $\mathrm{cDCs}$ may be affected by proinflammatory cytokines.

In this study, scrub typhus patients displayed dramatic drops in circulating $\mathrm{pDC}$ and $\mathrm{cDC}$ levels, and this trend reflected disease severity. Consistent with the present study, septic shock patients displayed reductions in both pDCs and cDCs, which were associated with the development of intensive care unit infection or fatal outcomes $(23,24)$. A model that used Citrobacter rodentium-treated pDC-depleted mice showed increased systemic inflammation, and polymicrobial sepsisinduced cDC-depleted mice demonstrated increased mortality, indicating that $\mathrm{pDCs}$ and $\mathrm{cDCs}$ play protective roles in bacterial infections $(25,26)$. Collectively, these results suggest that the depletion of both pDCs and cDCs is a feature of infectioninduced immune dysregulation, leading to the propagation and aggravation of systemic inflammation in scrub typhus infection.

Our data revealed enhanced co-stimulatory and co-inhibitory marker expression in both pDCs and cDCs. The expression of a co-stimulatory marker, indicated by CD86, was increased on pDCs during scrub typhus infection, which reflected their T-cell co-stimulatory function, consistent with previous studies on human immunodeficiency virus (HIV) and hepatitis $\mathrm{C}$ virus (HCV) infections $(27,28)$. In vitro experiments reported that $O$. tsutsugamushi-infected murine bone marrow-derived DCs or human moDCs exhibited increased CD86 expression $(17,18)$. Of note, the $\mathrm{CD} 86^{+} \mathrm{cDCs}$ were sustained at high levels regardless of the infection status, indicating that the T-cell priming function of cDCs was not solely dependent upon the scrub typhus infection. In contrast, the expression of a co-inhibitory marker, indicated by CD274, was higher in both pDCs and cDCs of scrub typhus patients than in those of HCs, in agreement with several studies on HIV and HCV infections $(27,28)$. Together, our findings suggest that both pDCs and cDCs expressed costimulatory and co-inhibitory molecules during scrub typhus infection, which could subsequently modulate the various immune reactions.

IFN- $\alpha$, TNF- $\alpha$, and IL-12 are well-known critical cytokines for controlling the intracellular growth of $O$. tsutsugamushi $(5,9$, $29,30)$. In the present study, the production of IFN- $\alpha$ and TNF$\alpha$ by pDCs upon CpG stimulation was found to be diminished in scrub typhus patients. In addition, following IFN- $\gamma / \mathrm{LPS}$ stimulation, cDCs exhibited the reduced production of IL-12 and TNF- $\alpha$ in scrub typhus patients. Consistent with our study, the production of these cytokines by $\mathrm{pDCs}$ and $\mathrm{cDCs}$ was decreased after stimulation with TLR 7/8 ligands in HIV 
A
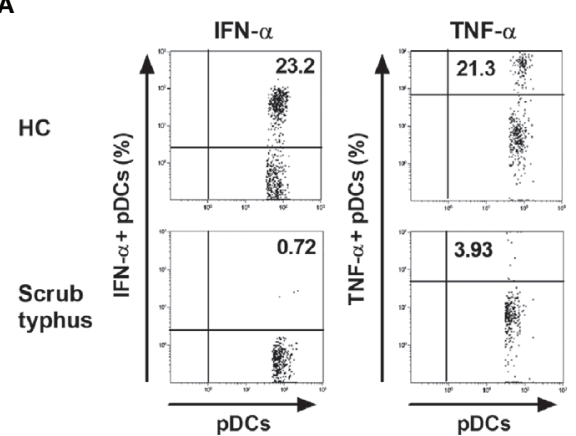

C

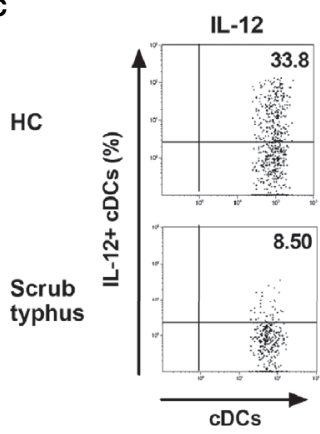

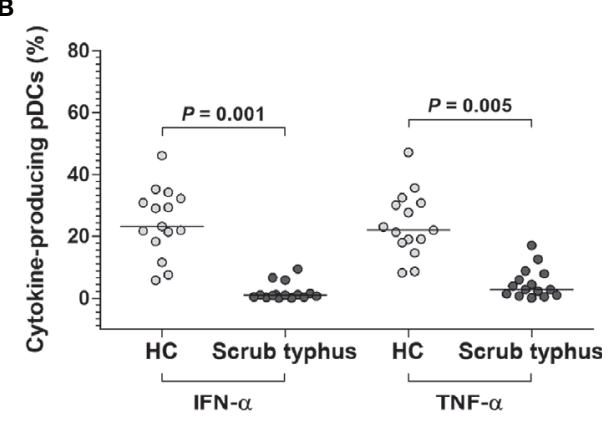

D

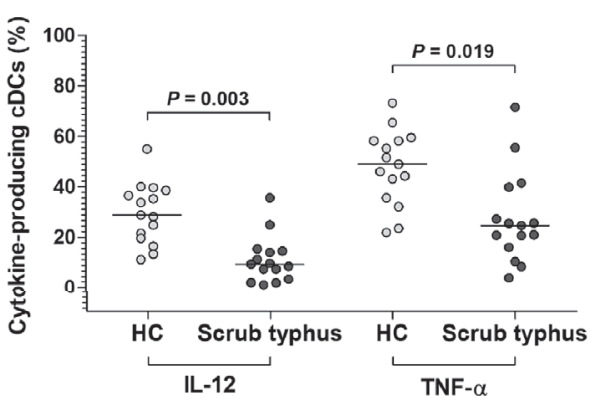

FIGURE 3 | Decreased expression of IFN- $\alpha$, IL-12, and TNF- $\alpha$ in pDCs and cDCs of scrub typhus patients. Freshly isolated PBMCs $\left(1 \times 10^{6} /\right.$ well) were incubated for 2 hours in the presence of $\mathrm{CpG}$ or the non-CpG ODN control for pDC stimulation, and in the presence of IFN- $\gamma$ and LPS for cDC stimulation. (A, C) Representative IFN- $\alpha$-, IL-12-, and TNF- $\alpha$-expressing pDCs or CDCs as determined by flow cytometry. The data in (B, D) were obtained from 15 HCs and 15 patients with scrub typhus. The symbols represent individual subjects and the horizontal lines are median values. $P$ values were calculated by ANCOVA test.

A

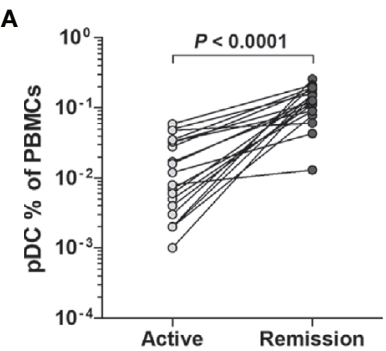

D

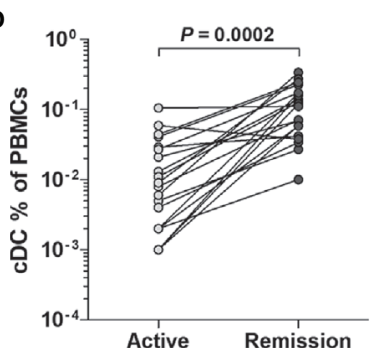

B

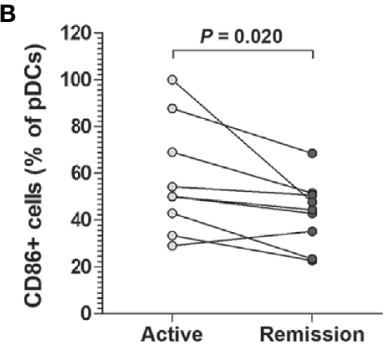

E

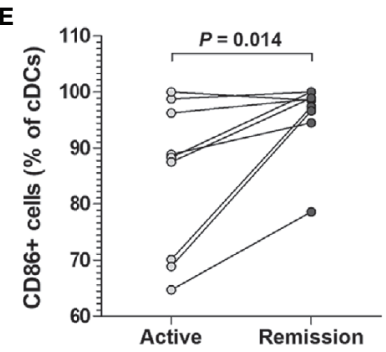

C
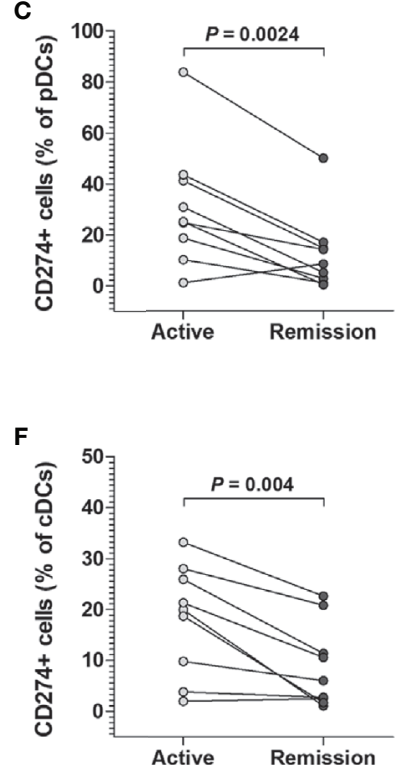

FIGURE 4 | Changes in cell percentages and surface phenotypes of circulating pDCs and cDCs from scrub typhus patients. The percentages of pDCs (A) and cDCs (D) in the peripheral blood of 20 scrub typhus patients during active and remission states were determined by flow cytometry. The percentages of CD86expressing (B, E), and CD274-expressing (C, F) pDCs and CDCs were determined by flow cytometry. The data in (B C, E, F) were obtained from nine patients with scrub typhus. The symbols represent individual subjects. $P$ values were calculated by Wilcoxon matched-pairs signed-rank test. 
A

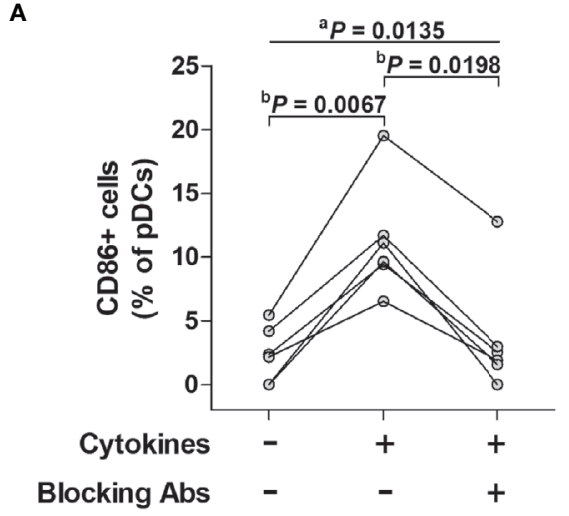

C

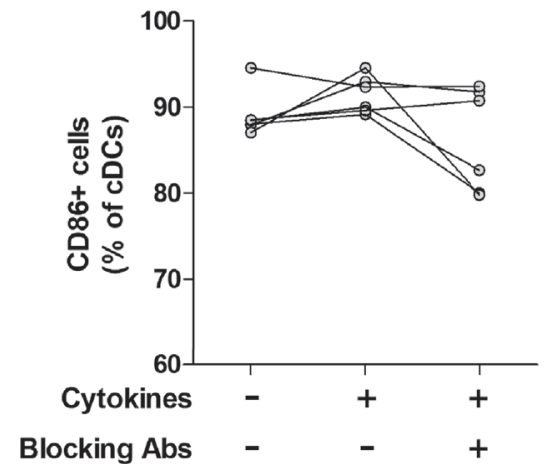

B

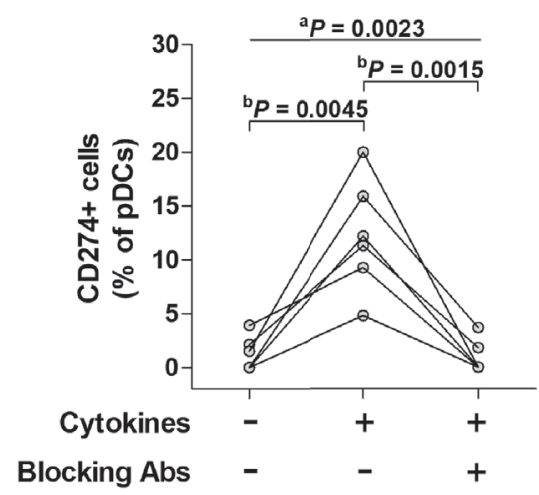

D

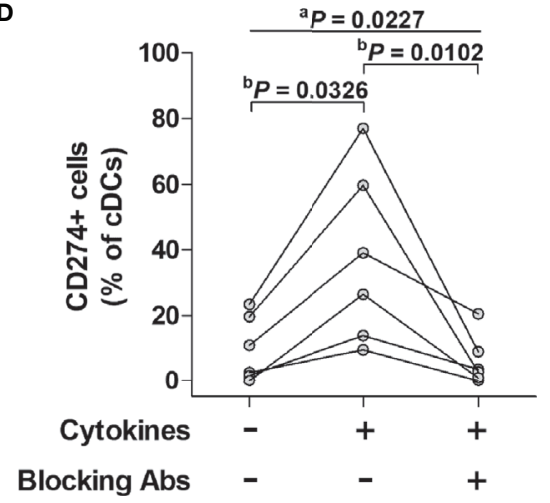

FIGURE 5 | Effect of a pro-inflammatory cytokine cocktail and its blocking antibody on surface phenotypes of pDCs and cDCs. Freshly isolated PBMCs were incubated for 24 hours in the presence or absence of cytokine inhibitors (i.e., blocking antibodies against a cocktail of IFN- $\alpha$, IL-12, and TNF- $\alpha$ and then stimulated with the cytokine cocktail for 16 hours. The stimulated cells were stained with APC-conjugated anti-CD11c, anti-CD86, and anti-CD274; BV421-conjugated anti-HLA-DR; FITC-conjugated Lineage Cocktail 1; PE-conjugated anti-CD86, anti-CD123, and anti-CD274 mAbs and then analyzed by flow cytometry.

(A, C) Percentage of CD86-expressing pDCs and CDCs. (B, D) Percentage of CD274-expressing pDCs and cDCs. The data were obtained from six HCs. ${ }^{2} P$ values among three groups were calculated by Kruskal-Wallis test. ${ }^{\mathrm{b}} \mathrm{P}$ values between two groups were calculated by Dunn's post hoc test.

infection $(27,31)$. In contrast, Chu et al. reported increased IL-12 and TNF- $\alpha$ secretion from $O$. tsutsugamushi-infected human moDCs (18). Interestingly, the TNF- $\alpha$ expression in monocytes varied from study to study according to the strains and dose of $O$. tsutsugamushi, the nature of the infected cell line or tissue, and animal or human models. For example, low-dose $O$. tsutsugamushi infection was found to induce the inhibition of a pro-inflammatory pathway as well as the up-regulation of an anti-inflammatory pathway, promoting bacterial replication, whereas high-dose infection reversed this response, enhancing bacterial clearance (5). One possible explanation for the decreased $\mathrm{pDC}$ and $\mathrm{cDC}$ cytokine function in scrub typhus patients is the delayed or impaired maturation of $\mathrm{pDC}$ and cDC during O. tsutsugamushi infection, suppressing immune function. Another explanation is the hypo-responsiveness of preactivated human DCs to in vitro restimulation.

Our data revealed that the proportion of circulating $\mathrm{pDCs}$ and cDCs were significantly correlated with disease severity in scrub typhus patients. Consistent with our data, previous studies showed that only blood $\mathrm{cDC}$ levels but not $\mathrm{pDC}$ levels were inversely correlated with the severity of dengue virus infection and severe fever with thrombocytopenia syndrome $(32,33)$. Compared to viral infections, however, little is known about the correlation between disease severity and the numbers of circulating pDCs or cDCs in other bacterial infections. Our novel observation revealed that the numerical depletion of both pDCs and $\mathrm{cDCs}$ was recovered during the remission state of the infection, with changes in co-stimulatory and co-inhibitory surface marker expression. The recovery of $\mathrm{pDC}$ and $\mathrm{cDC}$ numbers strongly supports our data reflecting the inversed correlation between those cell numbers and disease severity. Moreover, the recovery of circulating $\mathrm{pDC}$ numbers was accompanied by a decreased expression of CD86 and CD274, suggesting that the antigen presentation or tolerogenic function of pDCs might be limited during the acute phase of the infection. This phenomenon was also firmly supported by our data demonstrating that $\mathrm{pDC}$ numbers were positively correlated with albumin levels, known as a negative acute phase reactant marker. Similar to pDCs, the expression of CD274 on cDCs was also decreased in the remission state, but the expression of CD86 was increased. Unlike pDCs, the difference in the CD86 expression pattern in cDCs might be due to the constitutionally 
high expression of CD86 in cDCs. Taken together, our findings indicate that DC activation and depletion reflects the severity of scrub typhus infection.

We hypothesized that a pro-inflammatory cytokine-rich environment could affect the expression of co-stimulatory and co-inhibitory markers in pDCs and cDCs during a scrub typhus infection. Our previous study reported the increased levels of cytokines such as IFN- $\gamma$, IL-12, and TNF- $\alpha$ in the early stage of a scrub typhus infection (11). After reproducing the proinflammatory milieu using a cytokine cocktail including IFN- $\gamma$, IL-12, and TNF- $\alpha$, we observed the dynamics of CD86 and CD274 expression patterns in pDCs and cDCs, which was similar to our data in scrub typhus patients. These results suggest that the altered features of pDCs and cDCs might be secondary to the pro-inflammatory environment in the acute stage of the infection.

CpG-containing oligonucleotides (CpG ODNs) act on Tolllike receptor 9 (TLR9) that is expressed on B cells and pDCs to stimulate the innate immune system. Three types of stimulatory CpG ODNs have been identified: CpG-A, CpG-B, and CpG-C, which differ in their immune stimulatory activities. CpG-As are known to induce high production of type I IFN in pDCs but are not recognized in human B cells (34). Krug et al. have reported that CpG-A induced monocyte-derived DC-like phenotypes in PBMCs within 3 days but not such changes in purified monocytes (35). In the present study, however, CpG ODN 2336, a prototype of CpG-A, was used for culturing PBMCs for 2 hours, which was too short to induce significant changes in monocytes among PBMCs. Nonetheless, there are concerns that the cytokines that these cells produce may impact our DCs so in reality. It would have been optimal to sort the DCs prior to treatment.

There are some limitations in the present study. Based on recent advances through the emergence of powerful single-cell RNA sequencing and deep phenotyping technologies, originally three key DC subsets ( $\mathrm{CDC} 1, \mathrm{cDC} 2$, and $\mathrm{pDC}$ ) in peripheral blood have been expanded to six putative subsets ( $\mathrm{cDC} 1, \mathrm{cDC} 2-$ $\mathrm{A}, \mathrm{cDC} 2-\mathrm{B}, \mathrm{CD} 16^{+} \mathrm{DC}, \mathrm{Axl}^{+} \mathrm{DC}$, and $\mathrm{pDC}$ ) (36). In the present study, the antibody panels used for classification of circulating DCs comprised Lineage cocktail 1, CD123, HLA-DR, and CD11c, limiting our ability to distinguish among $\mathrm{CDC}$ subtypes or between $\mathrm{pDCs}$ and $\mathrm{Axl}^{+}$DCs. CD11c positivity has been known as the marker selecting cDCs. Circulating Lin'HLA$\mathrm{DR}^{\text {high }} \mathrm{CD} 11 \mathrm{c}^{+}$cells were defined as $\mathrm{cDCs}$. By selecting CD11c, we may have excluded a subset of $\mathrm{CDCs}$ which are CD11 $\mathrm{c}^{\text {low }}$ and are typically about $10 \%$ of total cDCs. This subset was mostly presumed to be $\mathrm{CDC1}$, according to human blood and tissue dendritic cell phenotypes described by Rhodes et al. (36). Furthermore, $\mathrm{CD}^{+} 6^{+} \mathrm{DC}$ reported newly by Villani et al. also expressed CD11c (37). Some lineage marker panels included only CD3, CD19 and CD56, whereas ours additionally included $\mathrm{CD} 14$ and CD16. Thus, both classical $\left(\mathrm{CD} 14^{++} \mathrm{CD} 16^{-}\right)$and nonclassical $\left(\mathrm{CD} 14^{+} \mathrm{CD} 16^{++}\right)$monocytes as well as T cells, B cells, and NK cells were negatively selected by our lineage panel. It is presumed that all monocyte subsets as well as $\mathrm{CD}_{16}{ }^{+} \mathrm{DCs}$ might have been excluded from CD $11 c^{+} \mathrm{cDCs}$ in the present study. Moreover, a number of computational flow cytometry tools such as FlowSOM, tSNE, oneSENSE, and ISOMAP have been developed to scale and represent high dimensional data of multiparametric flow cytometry (38). This approach could provide unbiased mapping and discovery of new cell phenotypes different from ones here identified using sequential manual gating. Further studies are warranted to answer this issue using unbiased gating strategy.

There are two methods for enumeration of absolute numbers of circulating DCs: dual-platform and single-platform. The dualplatform method is to calculate absolute numbers of circulating DCs by multiplying the percent amount of DCs in the mononuclear cells gate (using a flow cytometer) by absolute PBMC count determined using a standard hemocytometer. However, calculating absolute counts based on isolated PBMCs is not reliable, because PBMC isolations vary significantly from batch to batch. In contrast, the single-platform method, which is more accurate and easier than the former, is to enumerate absolute numbers of circulating DCs directly in a true count tube containing flow differential beads using a flow cytometer without hemocytometer. Unfortunately, the dual-platform method was used in the present study. The strategy used for quantification of absolute numbers is also to measure a fraction per volume (a relative metric).

In conclusion, this study firstly demonstrated that circulating pDCs and cDCs were numerically deficient and functionally impaired in scrub typhus patients. In addition, reduced $\mathrm{CDC}$ and $\mathrm{pDC}$ numbers reflected disease severity. We reported the novel finding that alterations in the expression levels of surface phenotypes of pDCs and cDCs could be affected by proinflammatory cytokines. These findings provide important insight into the dynamics of DC responses, which could present useful clues for future immunotherapy or vaccine development.

\section{DATA AVAILABILITY STATEMENT}

The original contributions presented in the study are included in the article/supplementary material. Further inquiries can be directed to the corresponding authors.

\section{ETHICS STATEMENT}

The study protocol was approved by the Institutional Review Board of Chonnam National University Hospital. Written informed consent was obtained from all participants in accordance with the Declaration of Helsinki.

\section{AUTHOR CONTRIBUTIONS}

S-JiK, K-JP, H-MJ, Y-NC, TO, SK, UK, K-HP, S-IJ, T-OK, HK, Y-GJ, JJ, S-JuK, and Y-WP designed this study, collected clinical information, analyzed raw data, performed statistical analysis, and contributed to writing of the paper. S-JiK, K-JP, H-MJ, and 
Y-NC performed experiments. All authors contributed to the article and approved the submitted version.

\section{FUNDING}

This work was supported by the National Research Foundation of Korea (2019R1A2C1003238, 2019R1I1A1A01040762), the Korea Health Technology R\&D Project through the Korea Health Industry Development Institute funded by the Ministry of Health \& Welfare, Republic of Korea (HI20C0079) and the

\section{REFERENCES}

1. Xu G, Walker DH, Jupiter D, Melby PC, Arcari CM. A Review of the Global Epidemiology of Scrub Typhus. PloS Negl Trop Dis (2017) 11(11):e0006062. doi: 10.1371/journal.pntd.0006062

2. Taylor AJ, Paris DH, Newton PN. A Systematic Review of Mortality From Untreated Scrub Typhus (Orientia Tsutsugamushi). PloS Negl Trop Dis (2015) 9(8):e0003971. doi: 10.1371/journal.pntd.0003971

3. Huang MH, Juan YH, Chen YT. Prolonged Coma in a Scrub Typhus Patient. Int J Infect Dis (2018) 77:5-7. doi: 10.1016/j.ijid.2018.09.015

4. Park SW, Ha NY, Ryu B, Bang JH, Song H, Kim Y, et al. Urbanization of Scrub Typhus Disease in South Korea. PloS Negl Trop Dis (2015) 9(5): e0003814. doi: 10.1371/journal.pntd.0003814

5. Diaz FE, Abarca K, Kalergis AM. An Update on Host-Pathogen Interplay and Modulation of Immune Responses During Orientia Tsutsugamushi Infection. Clin Microbiol Rev (2018) 31(2):e00076-17. doi: 10.1128/CMR.00076-17

6. Moron CG, Popov VL, Feng HM, Wear D, Walker DH. Identification of the Target Cells of Orientia Tsutsugamushi in Human Cases of Scrub Typhus. Mod Pathol (2001) 14(8):752-9. doi: 10.1038/modpathol.3880385

7. Paris DH, Phetsouvanh R, Tanganuchitcharnchai A, Jones M, Jenjaroen K, Vongsouvath $\mathrm{M}$, et al. Orientia Tsutsugamushi in Human Scrub Typhus Eschars Shows Tropism for Dendritic Cells and Monocytes Rather Than Endothelium. PloS Negl Trop Dis (2012) 6(1):e1466. doi: 10.1371/journal. pntd.0001466

8. Soong L. Dysregulated Th1 Immune and Vascular Responses in Scrub Typhus Pathogenesis. J Immunol (2018) 200(4):1233-40. doi: 10.4049/ jimmunol.1701219

9. Min CK, Kim HI, Ha NY, Kim Y, Kwon EK, Yen NTH, et al. A Type I Interferon and IL-10 Induced by Orientia Tsutsugamushi Infection Suppresses Antigen-Specific T Cells and Their Memory Responses. Front Immunol (2018) 9:2022. doi: 10.3389/fimmu.2018.02022

10. Kang SJ, Jin HM, Won EJ, Cho YN, Jung HJ, Kwon YS, et al. Activation, Impaired Tumor Necrosis Factor-Alpha Production, and Deficiency of Circulating Mucosal-Associated Invariant T Cells in Patients With Scrub Typhus. PloS Negl Trop Dis (2016) 10(7):e0004832. doi: 10.1371/ journal.pntd.0004832

11. Kang SJ, Jin HM, Cho YN, Kim SE, Kim UJ, Park KH, et al. Increased Level and Interferon-Gamma Production of Circulating Natural Killer Cells in Patients With Scrub Typhus. PloS Negl Trop Dis (2017) 11(7):e0005815. doi: 10.1371/journal.pntd.0005815

12. Kang SJ, Jin HM, Cho YN, Oh TH, Kim SE, Kim UJ, et al. Dysfunction of Circulating Natural Killer T Cells in Patients With Scrub Typhus. J Infect Dis (2018) 218(11):1813-21. doi: 10.1093/infdis/jiy402

13. Balan S, Saxena M, Bhardwaj N. Dendritic Cell Subsets and Locations. Int Rev Cell Mol Biol (2019) 348:1-68. doi: 10.1016/bs.ircmb.2019.07.004

14. Wculek SK, Cueto FJ, Mujal AM, Melero I, Krummel MF, Sancho D. Dendritic Cells in Cancer Immunology and Immunotherapy. Nat Rev Immunol (2020) 20(1):7-24. doi: 10.1038/s41577-019-0210-Z

15. Bakdash G, Schreurs I, Schreibelt G, Tel J. Crosstalk Between Dendritic Cell Subsets and Implications for Dendritic Cell-Based Anticancer Immunotherapy. Expert Rev Clin Immunol (2014) 10(7):915-26. doi: 10.1586/1744666X.2014.912561
Chonnam National University Hospital Biomedical Research Institute (CRI18042-21).

\section{ACKNOWLEDGMENTS}

We thank all subjects who participated in this study. We would like to especially thank Ms. Hyun-Ju Jung, Jiwon Jeon, Sehyeon Ji, and Ah Young Kim for their support in facilitating patient recruitment at Chonnam National University Hospital, Gwangju, Republic of Korea.

16. Swiecki M, Colonna M. The Multifaceted Biology of Plasmacytoid Dendritic Cells. Nat Rev Immunol (2015) 15(8):471-85. doi: 10.1038/nri3865

17. Choi JH, Cheong TC, Ha NY, Ko Y, Cho CH, Jeon JH, et al. Orientia Tsutsugamushi Subverts Dendritic Cell Functions by Escaping From Autophagy and Impairing Their Migration. PloS Negl Trop Dis (2013) 7(1): e1981. doi: 10.1371/journal.pntd.0001981

18. Chu H, Park SM, Cheon IS, Park MY, Shim BS, Gil BC, et al. Orientia Tsutsugamushi Infection Induces CD4+ T Cell Activation Via Human Dendritic Cell Activity. J Microbiol Biotechnol (2013) 23(8):1159-66. doi: 10.4014/jmb.1303.03019

19. Kim IS, Seong SY, Woo SG, Choi MS, Kang JS, Chang WH. Rapid Diagnosis of Scrub Typhus by a Passive Hemagglutination Assay Using Recombinant 56-Kilodalton Polypeptides. J Clin Microbiol (1993) 31(8):2057-60. doi: 10.1128/JCM.31.8.2057-2060.1993

20. Astrup E, Janardhanan J, Otterdal K, Ueland T, Prakash JA, Lekva T, et al. Cytokine Network in Scrub Typhus: High Levels of Interleukin-8 Are Associated With Disease Severity and Mortality. PloS Negl Trop Dis (2014) 8(2):e2648. doi: 10.1371/journal.pntd.0002648

21. Pacanowski J, Kahi S, Baillet M, Lebon P, Deveau C, Goujard C, et al. Reduced Blood CD123+ (Lymphoid) and CD11c+ (Myeloid) Dendritic Cell Numbers in Primary HIV-1 Infection. Blood (2001) 98(10):3016-21. doi: 10.1182/ blood.v98.10.3016

22. Marin E, Cuturi MC, Moreau A. Tolerogenic Dendritic Cells in Solid Organ Transplantation: Where Do We Stand? Front Immunol (2018) 9:274. doi: 10.3389/fimmu.2018.00274

23. Grimaldi D, Louis S, Pene F, Sirgo G, Rousseau C, Claessens YE, et al. Profound and Persistent Decrease of Circulating Dendritic Cells Is Associated With ICU-Acquired Infection in Patients With Septic Shock. Intensive Care Med (2011) 37(9):1438-46. doi: 10.1007/s00134-011-2306-1

24. Guisset O, Dilhuydy MS, Thiebaut R, Lefevre J, Camou F, Sarrat A, et al. Decrease in Circulating Dendritic Cells Predicts Fatal Outcome in Septic Shock. Intensive Care Med (2007) 33(1):148-52. doi: 10.1007/s00134-006-0436-7

25. Rahman T, Brown AS, Hartland EL, van Driel IR, Fung KY. Plasmacytoid Dendritic Cells Provide Protection Against Bacterial-Induced Colitis. Front Immunol (2019) 10:608. doi: 10.3389/fimmu.2019.00608

26. Scumpia PO, McAuliffe PF, O’Malley KA, Ungaro R, Uchida T, Matsumoto T, et al. CD11c+ Dendritic Cells Are Required for Survival in Murine Polymicrobial Sepsis. J Immunol (2005) 175(5):3282-6. doi: 10.4049/jimmunol.175.5.3282

27. Cavaleiro R, Baptista AP, Soares RS, Tendeiro R, Foxall RB, Gomes P, et al. Major Depletion of Plasmacytoid Dendritic Cells in HIV-2 Infection, an Attenuated Form of HIV Disease. PloS Pathog (2009) 5(11):e1000667. doi: 10.1371/journal.ppat.1000667

28. Shen T, Chen X, Chen Y, Xu Q, Lu F, Liu S. Increased PD-L1 Expression and PD-L1/CD86 Ratio on Dendritic Cells Were Associated With Impaired Dendritic Cells Function in HCV Infection. J Med Virol (2010) 82(7):11529. doi: 10.1002/jmv.21809

29. Geng P, Jerrells TR. The Role of Tumor Necrosis Factor in Host Defense Against Scrub Typhus Rickettsiae. I. Inhibition of Growth of Rickettsia Tsutsugamushi, Karp Strain, in Cultured Murine Embryonic Cells and Macrophages by Recombinant Tumor Necrosis Factor-Alpha. Microbiol Immunol (1994) 38(9):703-11. doi: 10.1111/j.1348-0421.1994.tb01845.x

30. Jerrells TR, Geng P. The Role of Tumor Necrosis Factor in Host Defense Against Scrub Typhus Rickettsiae. II. Differential Induction of Tumor Necrosis Factor- 
Alpha Production by Rickettsia Tsutsugamushi and Rickettsia Conorii. Microbiol Immunol (1994) 38(9):713-9. doi: 10.1111/j.1348-0421.1994.tb01846.x

31. Huang J, Yang Y, Al-Mozaini M, Burke PS, Beamon J, Carrington MF, et al. Dendritic Cell Dysfunction During Primary HIV-1 Infection. J Infect Dis (2011) 204(10):1557-62. doi: 10.1093/infdis/jir616

32. Lertjuthaporn S, Khowawisetsut L, Keawvichit R, Polsrila K, Chuansumrit A, Chokephaibulkit K, et al. Identification of Changes in Dendritic Cell Subsets That Correlate With Disease Severity in Dengue Infection. PloS One (2018) 13 (7):e0200564. doi: 10.1371/journal.pone.0200564

33. Zhang W, Li M, Xiong S, Wang H, Xiong Y, Li M, et al. Decreased Myeloid Dendritic Cells Indicate a Poor Prognosis in Patients With Severe Fever With Thrombocytopenia Syndrome. Int J Infect Dis (2017) 54:113-20. doi: 10.1016/ j.ijid.2016.11.418

34. Hartmann G, Battiany J, Poeck H, Wagner M, Kerkmann M, Lubenow N, et al. Rational Design of New CpG Oligonucleotides That Combine B Cell Activation With High IFN-Alpha Induction in Plasmacytoid Dendritic Cells. Eur J Immunol (2003) 33(6):1633-41. doi: 10.1002/eji.200323813

35. Krug A, Rothenfusser S, Selinger S, Bock C, Kerkmann M, Battiany J, et al. CpG-A Oligonucleotides Induce a Monocyte-Derived Dendritic Cell-Like Phenotype That Preferentially Activates CD8 T Cells. J Immunol (2003) 170 (7):3468-77. doi: 10.4049/jimmunol.170.7.3468
36. Rhodes JW, Tong O, Harman AN, Turville SG. Human Dendritic Cell Subsets, Ontogeny, and Impact on HIV Infection. Front Immunol (2019) 10:1088. doi: 10.3389/fimmu.2019.01088

37. Villani AC, Satija R, Reynolds G, Sarkizova S, Shekhar K, Fletcher J, et al. Single-Cell RNA-Seq Reveals New Types of Human Blood Dendritic Cells, Monocytes, and Progenitors. Science (2017) 356(6335):eaah4573. doi: $10.1126 /$ science.aah4573

38. Collin M, Bigley V. Human Dendritic Cell Subsets: An Update. Immunology (2018) 154(1):3-20. doi: 10.1111/imm.12888

Conflict of Interest: The authors declare that the research was conducted in the absence of any commercial or financial relationships that could be construed as a potential conflict of interest.

Copyright (๑) 2021 Kang, Park, Jin, Cho, Oh, Kim, Kim, Park, Jung, Kim, Kim, Jo, Ju, Kee and Park. This is an open-access article distributed under the terms of the Creative Commons Attribution License (CC BY). The use, distribution or reproduction in other forums is permitted, provided the original author(s) and the copyright owner(s) are credited and that the original publication in this journal is cited, in accordance with accepted academic practice. No use, distribution or reproduction is permitted which does not comply with these terms. 